Historic, Archive Document

Do not assume content reflects current scientific knowledge, policies, or practices. 

Wholesale Prices of

\section{California Bulbs}

Season

1913

\section{CARL PURDY}

Ukiah. California, U. S. A. 
Terms: All prices, with the exception of those for Lilies, Trilliums, and Miscellaneous Plants, are for delivery free to customer by post or express at our option.

Bills are due 90 days from date, with 3 per cent for cash.

I guarantee safe delivery and good quality. Boxes and packing are not charged to customer. By freight I would allow the difference between expressage and freight to customer, but freight has been very slow of recent years, and I will not assume any risk of loss by îreight shipments.

Ukiah is a railroad, express, telephone, and international money-order office. My cable address is registered, "Purdy, Ukiah."

25 at 100 rate; 250 at 1000 rate.

\section{BRODIA EAS}

$100 \quad 1000$

Capitata, Blue.......... $\$ 1.50 \$ 10.00$

Coccinea, Floral Fire-

cracker, $1-2$ to $5-8$ in..... 2.25

Coccinea, $5-8$ to 1 in...... 3.00

Coccinea, Giant Bulbs..... 6.00

Grandiflora, Blue........ 1.50

-Ixoides Splendens......... 2.25

Laxa, Blue in Umbels....... 1.50

Laxa, Purple King......... 4.50

Lactea, Milky White....... 1.50

Peduncularis, White....... 2.25

Purdyii ............... 3.00

Stellaris............... 2.25

Volubis, $5-8$ inch......... 3.00

Volubis, $5-8$ to 1 inch....... 4.50

Volubis, 1 inch, Giants..... 6.00

Bloomeria Aurea........... 2.2515 .00

CALOCHORTUS (Globe Tulips)

Albus .................\$1.50 \$15.00

Albus, The Pearl.......... 2.25

Amabilis .............. 1.50

-Amoenus............. 4.50

Pulchellus True......... 4.50

\section{CALOCHORTUS (Star Tulips)}

Benthamii............\$1.50

Lilacinus . . . . . . . . . . . 1.50

Maweanus Major......... 1.50

Purdyii .............. 3.00
CALOCHORTUS (Mariposa Tulips)

1001000

-Catalina............\$6.00

Luteus ................ $2.25 \quad 15.00$

Venustus Citrinus......... 2.2515 .00

Venustus, El Dorado Strain... 2.2515 .00

-Venustus, El Dorado Gold

Blotched .............6. 6.00

15.00 Venustus, El Dorado, Red

to Pink...............4 450

Venustus, El Dorado, White. . $3.00 \quad 20.00$

Venustus Oculatus......... 2.2515 .00

Venustus Purpurascens..... $3.00 \quad 20.00$

Venustus Roseus........... $3.00 \quad 20.00$

Vesta, Large Bulbs. . . . . . . $4.50 \quad 30.00$

Vesta, Good Bulbs......... $3.00 \quad 20.00$

CALOCHORTUS

(Various Large Mariposa Tulips)

-Clavatus Ventura....... \$10.00

Gunnisonii ............. 4.50

Howellii, rare........... 10.00

Macrocarpus ........... 4.50

Leichtlinii (September

delivery) . . .......... $3.00 \quad 20.00$

Nitidus .............. 2.25

-Plummerae...........6.6.00

Splendens ............. $4.50 \quad 20.00$

10.00 Splendens Rubra......... 4.50

$10.00 \quad$ Nuttallii ................ 3.00

10.00 MIXED CALOCHORTUS

Five or six varieties......\$1.50 10.00

\footnotetext{
-Items marked with a hyphen are very uncertain. In July we will send out a bulletin stating which of these has been secured.
} 
Mixed Mariposas alone...... $1.75 \quad 14.00$

Mixed Clobe Tulips.......... $1.75 \quad 14.00$

Mixed Star Tulips.......... $1.75 \quad 14.00$

Mixed El Dorado, Fine..... 2.25 15.00

\section{CAMASS̊IAS}

Cusickii..............\$6.00

Esculenta, Improved....... 75

Leichtliniı, Purple........ 3.00

Leichtlinii, Cream......... 3.00

Leichtlinii Montana....... 3.00

\section{ERYTHRONIUM}

Grandiflorum or Nuttalianum.$\$ 3.00 \$ 20.00$

Grandiflorum Robustum..... . 3.00

Citrinum ................. 3.00

Californicum............. 150

Californicum, White Beautp. . 300

Giganteum ............. 3.00

Hartweggii ............ 1.50

Howellii, rare........... 4.50

Hendersonii ............. 2.25

Revolutum Type.......... 3.00

Revolutum Praecox....... 3.00

Revolutum Johnsonii...... 4.50

Revolutum, Pink Beauty.... 4.50

Purpurascens ........... 3.00

Revolutum Watsonii is E. Giganteum.

\section{FRITILLARIAS}

Agrestis .............. $\$ 4.50$

Liliacea.............. 4.50

Pluriflora............. 4.50

\section{Tall Varieties}

Coccinea ...............\$1.50 $\$ 30.00$

Lanceolata............ 4.50

Lanceolata Gracilis........ 4.50

Recurva, 3-4 to 1 inch...... 2.25

Recurva, 1 to $11-4$ inch.... 4.50

Recurva, 11-4 to 1 1-2 inch. . 5.00

Recurva, Giants, 1 1-2 in. up 10.00

\section{CALIFORNIA LILIES \\ Humboldtii Group}

Humboldtii Type, 7 tก 8 in.. $\$ 9.00 \$ 7 j .00$

Humboldtii Type, 8 to 9 in...12.00 100.00

Humboldtii Type, 9 to 11 in. .20.00
Humboldtii Magnificum

largest ...............30.00

Humboldtti Magnificum, good .20 .00

Bloomerianum............. 12.50

Columbianum, large.......12.50

Columbianum, good....... 7.50

Bolanderii............ 20.00

Washingtonianum Group

Washingtonianum Purpureum

7 to 8 inches...........\$9.00 $\$ 75.00$

Washingtonianum Purpureum

8 to 9 inches ........... 12.00

Washingtonianum Purpureum,

9 to 11 inches.........20.00

In the garden Lilium $W^{*}$ ashingtonianum grows smailer and more compact and seldom larger than the 7 to 8 inch size. These can be supplied at the price of the 8 to 9 inch collected. Supply is at present limited.

15.00 Rubescens (late October deliv20.00 ery, as they do not carry well earlier ...............20.00

Kelloggii, very rare and well marked ............20.00

\section{Pardalinum Group}

Pardalinum, very heavy .....\$6.00

Pardalinum, Fine Single

Eyes................ 4.50

Pardalinum, good........ 3.00

Roezlii................12.50

Parryii, Large Bulbs....... 30.00

Parryil, Good Bulbs.........20.00

Pardalinum Group (Small Bog Lilies)

Parvum, very large.......20.00

Parvum, good.............12.50

Parvum Luteum...........12.50

Parviflorum ............. 7.50

Maritimum............20.00

\section{TRILLIUMS}

Ovatum..............\$3.00

Rivale ............... 6.00

Sessile Californicum....... $4.50 \quad 30.00$

Sessile, Snow Queen....... $4.50 \quad 30.00$ 
Sessille Rubra, Deep Ma- the brightest of spring flowers. The roots roon. Supply now sure $4.50 \quad 30.00$ are like a carrot and fleshy and can be MISCELLANEOUS PLANTS DODECATHEONS OR AMERICAN COWSLIPS

Hendersonii .............\$3.00

Clevelannii .............. $\$ .00$

Patulum, Rose.......... 4.50

TERRESTRIAL ORCHIDS

Cypripedium Californicum . . \$12.50

C. Fasciculatum ......... 18.00

Montanum............ 10.00

Epipactes Gigantea....... 7.50

OENOTHERA OVATA

handled dry. A fine novelty.

Price...............\$5.00

\section{LEWISIAS}

Tweedii, wonderfully fine. . .\$30.00

Howellii, True; doz. $\$ 3 \ldots . .20 .00$

Cotyledon, rare. Supply sure.

Doz. $\$ 3 . \ldots \ldots \ldots \ldots \ldots .20 .00$

Oppositifolia........... 7.50

Rediviva............ 3.00

Leeana .............. 20.00

- DARLINGTONIAS OR PITCHER PLANTS

I can supply fine roots from September

A beautiful primrose having circles of leaves flat on the ground and a great number of bright yellow flowers. One of

to April at $\$ 12.50$ per 100 . Buyer must pay expressage and can have them of any size desired.

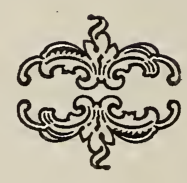

